\title{
Insertion Des Jeunes Déscolarisés Dans La Médecine Traditionnelle En Côte d'Ivoire : Activation Des Ressources Relationnelles Et Ideologiques Comme Strategie
}

\section{Adrien Kouakou Koua}

Doctorant,Institut des Sciences Anthropologiques de Développement (ISAD) Université Félix Houphouët Boigny de Cocody-Abidjan ; Côte d’Ivoire

\section{Léopold Yao Yao,} Gérard Kouakou N'Goran

Enseignant-chercheur

Institut des Sciences Anthropologiques de Développement (ISAD) Université Félix Houphouët Boigny de Cocody-Abidjan Côte d’Ivoire

\section{Abstract}

This study highlights the resources mobilized by school youth for their inclusion in the activities of traditional medicine. It presents the results of a survey conducted in the district of Abidjan in 2015, with a target population of 223 actors of traditional medicine and mobilizes interactionnist strategic analysis to make sense of the phenomenon under study. Thus it is clear from this study that the school youth encountered in this activity, mostly have primary education (60.54\%), and to a lesser extent the secondary level (32.63\%). They are also, traditional medicine sellers; and mostly professional experience of at least 5 years and over (65.92\%). For inclusion in the traditional therapy, it appears that these have recourse to relational resources, as ideological resources or ideological productions. Thus, these resources together, are proving highly strategic for their integration into traditional therapy.

Keywords: Insert, youth school, medicine, relational resources, ideological resources strategy

\section{Résumé}

La présente étude met en exergue les ressources mobilisées par les jeunes déscolarisés pour leur insertion dans les activités de la médecine traditionnelle. Elle présente les résultats d'une enquête réalisée dans le 
district d’Abidjan en 2015, auprès d'une population cible de 223 acteurs de la médecine traditionnelle et mobilise l'analyse stratégique interactionniste pour donner sens au phénomène étudié. Ainsi il ressort de cette étude, que les jeunes déscolarisés rencontrés dans cette activité, ont majoritairement le niveau primaire (60.54\%), et dans une moindre mesure le niveau secondaire (32,63\%). Ils sont également, vendeurs de médicaments traditionnels ; et ont en majeure partie une expérience professionnelle d'au moins 5 ans et plus (65.92\%). Pour leur insertion dans la tradithérapie, il ressort que ceux-ci ont recours aux ressources relationnelles, tout comme aux ressources idéologiques ou productions idéologiques. Ainsi, ces ressources, dans leur ensemble, se révèlent hautement stratégiques pour leur insertion dans la tradithérapie.

Mots clés: Insertion, Jeunes déscolarisés, Médecine traditionnelle, Ressource relationnelle, Ressources idéologiques, Stratégie

\section{Introduction}

Lorsqu'on évoque la genèse de l’exercice de la médecine traditionnelle africaine en tant qu'activité, la mémoire collective s’ouvre sur les personnes âgées et sur le caractère ésotérique qui fondent la geste de ces acteurs. Ainsi depuis des siècles, l'exercice de la médecine traditionnelle africaine a toujours été attribué à des personnes âgées ne sachant ni lire ni écrire. Cette situation de monopole (constatée) des personnes âgées, dans la pratique de la médecine traditionnelle africaine, amène à dire que « la civilisation traditionnelle africaine produit des hommes conformes à un modèle social où l’âge de l’individu joue un rôle prépondérant » (Giresca, 1995). A ce propos Faye (2001) fait remarquer que « les personnes âgées sont plus réceptives à la médecine traditionnelle alors que les jeunes, à cause de l'école ont le mépris de la tradition ». Il n’est donc pas surprenant que certains travaux à caractère sociodémographique sur la médecine traditionnelle en Afrique situent l'âge du tradipraticien entre 29 et 90 ans (Kroa, 2000 ; Yangni Angate, 2004 ; Coulibaly, 2007). En effet, ces travaux, dans leur ensemble, font remarquer que la plupart des tradipraticiens enquêtés appartiennent à la tranche d’âge de 50 à 90 ans et ne savent ni lire, ni écrire.

Aujourd'hui, les médias, les ONG et les organismes de recherche scientifique dressent un tableau rajeuni des ressources humaines de la médecine traditionnelle africaine. Plusieurs études à caractère sociodémographique sur la médicine traditionnelle africaine ont été diligentées par des laboratoires d'universités et de services du ministère de la santé. Selon Akoto et al (2001), dans le district sanitaire de Bafia (Cameroun), les tradipraticiens ont été en général scolarisés : seul un 
cinquième des tradipraticiens n'est jamais allé à l'école, plus de la moitié d'entre eux ont abandonné l'école au cours du cycle primaire, et plus du quart (27\%) a atteint au moins le niveau secondaire. En Côte d'Ivoire, selon l'étude de Manouan et al (2010), les tradipraticiens enquêtés dans le district d'Abidjan appartiennent pour la plupart à la tranche d’âge de 40 à 49 ans dans 29\% des cas ; et de 50 à 59 ans dans $24 \%$ des cas. Aussi, ajoutent-ils que l'âge minimum, et maximum de ceux-ci est compris respectivement entre 21 ans et 86 ans avec une moyenne d'âge de 46 ans. Selon cette même étude, $61 \%$ des tradipraticiens enquêtés ont au moins le niveau d'étude primaire. Cependant, cette recomposition du facteur humain ou des ressources humaines de la médecine traditionnelle en faveur des jeunes déscolarisés qui savent lire et écrire est à l'origine de l'émergence d'un conflit entre acteurs de la médecine traditionnelle. Ce conflit oppose d'un côté les jeunes déscolarisés qui ont décidé de donner une nouvelle image à la médecine traditionnelle ivoirienne ; et de l'autre côté les anciens, ou les plus âgés qui continuent d'exercer leurs dons sous ses formes traditionnelles, en perpétuant les conditions de leurs usages. Depuis lors il est reconnu que ce rapport conflictuel entre acteurs de la médecine traditionnelle prend des proportions stigmatisantes. Il convient donc de remarquer que ces conflits parfois ouverts ou dissimulés dans les représentations et pratiques thérapeutiques traditionnelles sont devenus récurrents de nos jours chez les thérapeutes traditionnels. A cet effet, Abdoumouleh (2009) souligne que « les anciens » manifestent ouvertement « leur hostilité » aux «jeunes guérisseurs », lesquels sont attirés par « le modernisme » et le profit. Il existe donc un réel problème à ce niveau qui nécessite que l'on utilise les ressources scientifiques pour comprendre cet environnement concurrentiel, qui semble favoriser l’insertion des jeunes déscolarisés dans les activités de la médecine traditionnelle, jadis dominées par les personnes âgées. Ce constat, nous amène à poser les questions suivantes: Quelles sont les caractéristiques socioprofessionnelles de ces jeunes déscolarisés ? Quelles sont les manœuvres ou stratégies mobilisées par ceux-ci, en vue de leur insertion dans la tradithérapie?

La présente étude vise donc, en dehors de l'identification des caractéristiques socioprofessionnelles de ces jeunes, à analyser les manœuvres et les stratégies adoptées par les jeunes déscolarisés en vue de s'affirmer, de s'adapter et de se maintenir dans l'exercice de l'activité de la médecine traditionnelle en Côte d’Ivoire.

\section{Methodologie De L'etude \\ Materiel}

A partir de la base de données des acteurs sociaux dont celle de la communauté des tradipraticiens d'une part; et d'autre part celle du 
Programme National de Promotion de la Médecine Traditionnelle (PNPMT) de Côte d'Ivoire, nous avons procédé à la sélection par un tirage aléatoire notre population d'enquête, composée d'acteurs de la médecine traditionnelle. Au nombre des critères retenus, nous mentionnons :

- $\quad$ être reconnu par le PNPMT;

- $\quad$ avoir l'âge qui varie de 15 à 43 ans, car selon les Nations unies (2005cité par Poison, 2009), l’âge médian, indicateur du degré de vieillissement d'une population, est plus faible en Afrique (19 ans) et varie de 15 à 43 ans selon les pays.

- $\quad$ être volontaire pour les entretiens, après avoir pris connaissance des objectifs de l'étude.

Des critères susmentionnés, 223 acteurs ont été identifiés, (tous tradipraticiens) et soumis à un questionnaire. En outre, des guides d'entretien ont été administrés aux autres acteurs de la médecine traditionnelle (services du PNPMT, Fédération Nationale de l'Association des Tradipraticiens).

\section{Methodes}

Il s'agit d'une étude transversale à visée descriptive et analytique qui combine les méthodes de recherche quantitative et qualitative. La population cible est constituée d'acteurs sociaux de la médecine traditionnelle reconnus comme tel par le PNPMT. L'enquête s'est déroulée en 2015 dans le district d'Abidjan. En effet, le champ social et culturel abidjanais en médecine traditionnelle très dynamique, est caractérisé par un système de relations concurrentielles et complémentaires entre acteurs et groupes pour exister et se faire reconnaître. En outre, les modes d'interactions entre ces acteurs sociaux de la médecine traditionnelle sont souvent d'ordre coopératif ou conflictuel et leurs rapports restent influencés par les intérêts ou les priorités et préoccupations. Ceux-ci sont définis aussi bien par les avantages économiques, récréatifs et culturels de la médecine traditionnelle que par ses obstacles d'ordre politique.

Ainsi, des guides d'entretien et un questionnaire leur ont été soumis. Ils ont porté sur le profil sociodémographique, les ressources relationnelles et idéologiques mobilisés par ces derniers.

Par ailleurs, l'étude de documents, de rapports relatifs aux activités du PNPMT et de certaines associations d'acteurs de la médecine traditionnelle nous a permis de vérifier et de recouper les informations recueillies par le biais d'un questionnaire et des guides d'entretien. Les données quantitatives obtenues ont été traitées statistiquement au moyen du logiciel statistique XL stat 2015 Microsoft avec le test de khi carré comme technique statistique appropriée pour tenir compte de la nature des données (les fréquences). L'utilisation du khi carrée de Person permet d'obtenir une valeur qui est significative au seuil de probabilité égale 0,05 et à un ddl. 
Quant à l'analyse qualitative des résultats, elle a privilégié l'analyse du contenu des sources documentaires et des informations récoltées auprès du Programme National de Promotion de la Médecine Traditionnelle et des propos des guérisseurs. L’interprétation des données recueillies a été reliée à l'analyse stratégique interactionniste.

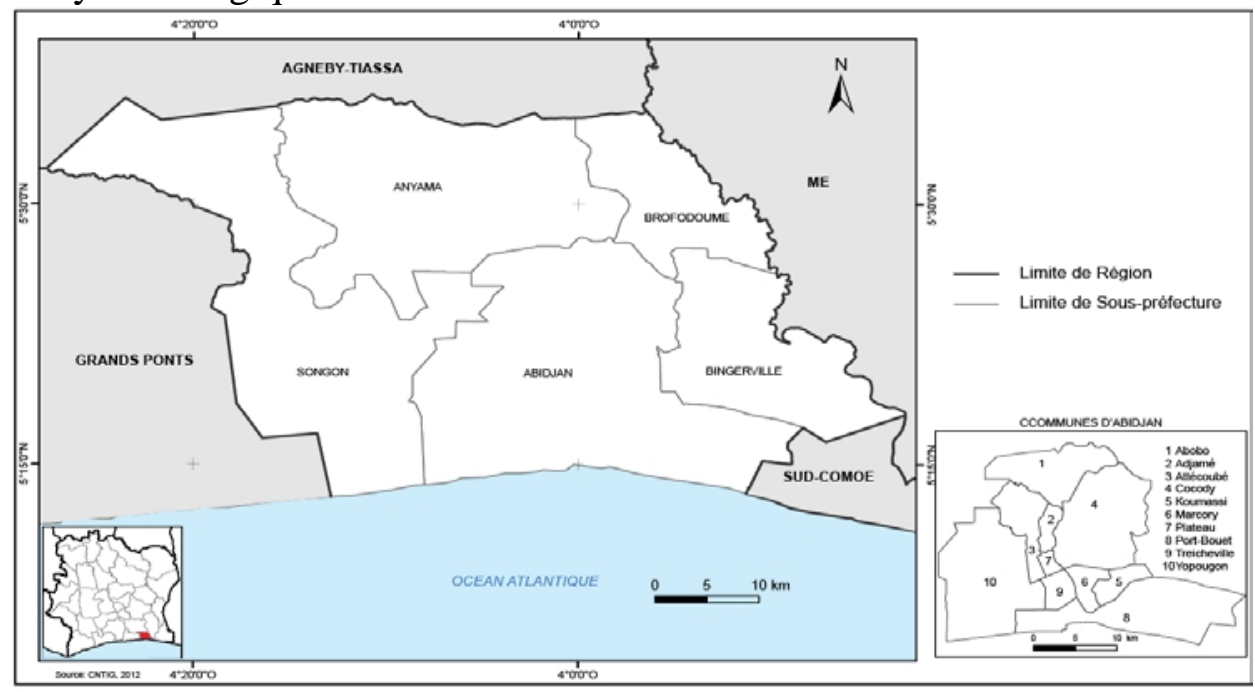

Figure. Le District Autonome d’Abidjan

Source : IGT, 2016

\section{Resultats}

Les Caracteristiques Socio-Professionnelles Des Jeunes Descolarisés De

\section{La Traditherapie}

Tableau $\mathbf{n}^{\circ} \mathbf{1}$ : Caractéristiques socioprofessionnelles des jeunes déscolarisés de la tradithérapie.

\begin{tabular}{ccc}
\hline NIVEAU D'ETUDE & EFFECTIF & $\%$ \\
\hline Primaire & 135 & $60.54 \%$ \\
Secondaire & 73 & $32.63 \%$ \\
Supérieur (universitaire) & 15 & $06.73 \%$ \\
\hline Total & 223 & $100 \%$ \\
\hline SITUATION PROFESSIONNELLE & & \\
\hline Guérisseurs & 61 & $27.35 \%$ \\
Vendeur & 116 & $52.02 \%$ \\
Démarcheur & 46 & $20.63 \%$ \\
\hline Total & 223 & $100 \%$ \\
\hline EXPÉRIENCE PROFESSIONNELLE & & \\
\hline Moins de cinq ans d'expérience & 76 & $34.08 \%$ \\
Cinq années d'expérience & 64 & $28.70 \%$ \\
Plus de cinq ans d'expérience & 83 & $37.22 \%$ \\
\hline Total & 223 & $100 \%$ \\
\hline
\end{tabular}

Source : Enquêtes personnelles, 2015 
A l'observation des caractéristiques socioprofessionnelles des jeunes déscolarisés de la tradithérapie, il ressort trois éléments majeurs qui ont retenus notre attention. Le niveau d'instruction, la situation professionnelle et l'expérience professionnelle des enquêtés. En ce qui concerne le niveau d'instruction, nous avons une prédominance du niveau primaire $60.54 \%$. Quant à la situation professionnelle, la majorité des enquêtés sont des vendeurs de médicaments traditionnels et représente $52.02 \%$ des enquêtés. Au niveau de l'expérience professionnelle, la majorité des enquêtés ont plus de cinq ans d'expérience et représente 37.22\% de l'ensemble des enquêtés. A partir de ces résultats on peut remarquer une mobilisation du capital humain par les jeunes déscolarisés à travers leur niveau d’instruction et leur expérience professionnelle.

\section{Les Ressources Relationnelles Mobilisées Par Les Jeunes Déscolarisés Dans l'espace Social De La Tradithérapie.}

Tableau $\mathbf{N}^{\circ} 2$ : Ressources relationnelles mobilisées en fonction de la situation socioprofessionnelle des enquêtés.

\begin{tabular}{|c|c|c|c|c|c|c|c|c|}
\hline \multirow{3}{*}{$\begin{array}{c}\text { RESSOURCES } \\
\text { RELATIONNELLES }\end{array}$} & \multicolumn{6}{|c|}{ SITUATION SOCIOPROFESSIONNELLE } & \multicolumn{2}{|c|}{ Total } \\
\hline & \multicolumn{2}{|c|}{ GUÉRISSEUR } & \multicolumn{2}{|c|}{ VENDEUR } & \multicolumn{2}{|c|}{ DÉMARCHEUR } & \multirow[b]{2}{*}{$\mathrm{N}$} & \multirow[b]{2}{*}{ (\%) } \\
\hline & $\mathrm{N}$ & $(\%)$ & $\mathrm{N}$ & $(\%)$ & $\mathrm{N}$ & $(\%)$ & & \\
\hline Les associations & 29 & (70.73) & 9 & $(21.95)$ & 3 & 7.32 & 41 & $\overline{100}$ \\
\hline Le parrainage & 9 & (16.07) & 29 & (51.79) & 18 & 32.14 & 56 & 100 \\
\hline La collaboration & 13 & (21.31) & 36 & 59.02 & 12 & 19.67 & 61 & 100 \\
\hline Familiale & 10 & (15.38) & 42 & 64.62 & 13 & 20 & 65 & 100 \\
\hline Total & 61 & (27.35) & 116 & 52.02 & 46 & 20.63 & 223 & 100 \\
\hline \multirow[t]{2}{*}{$\mathrm{P}$ value } & & & & & & $<0,0001$ & & \\
\hline & \multicolumn{3}{|c|}{ V de Cramer $=0,341$} & & $6 \mathrm{ddl}$ & & & \\
\hline
\end{tabular}

Les données du tableau nous renseignent sur les ressources relationnelles mobilisées par les acteurs de la médecine traditionnelle en fonction de leur situation professionnelle. En effet, il ressort de l'enquête que les guérisseurs représentent $70.73 \%$ de ceux qui ont mobilisé les associations comme ressource d'insertion dans la médecine traditionnelle. On constate que les vendeurs de médicaments quant à eux mobilisent l'accompagnement (64.62\%). Enfin, la ressource privilégiée par les démarcheurs est le parrainage soit 32.14\%. Dans l'ensemble, chaque acteur de la médecine traditionnelle enquêté, mobilise des ressources relationnelles différentes de celles des autres. Le test de $\chi^{2}$ a donc été effectué pour confirmer ou infirmer la mobilisation des différentes ressources relationnelle en fonction de la situation socioprofessionnelle des acteurs. Après le calcul le $\mathrm{P}$ value $\mathrm{du} \chi^{2}$, on obtient $\mathrm{P}$ égale 0,0001et le $\mathrm{V}$ de Cramer égale 0,341 avec le nombre de degré de liberté $(\mathrm{ddl})$ égal à 6 . Il existe une relation significative $(\mathrm{p}<0,05)$ 
entre les ressources utilisées et la situation socio professionnelle des enquêtés. En d’autres termes, les guérisseurs privilégient les associations par rapport aux autres ressources alors que les vendeurs de médicaments et les démarcheurs privilégient respectivement l’accompagnement et le parrainage.

\section{Les Ressources Idéologiques Mobilisées Par Les Jeunes Déscolarisés Dans L’activité De La Médecine Traditionnelle}

Tableau N³ : Ressources idéologiques mobilisées en fonction de la situation socioprofessionnelle des enquêtés.

\begin{tabular}{|c|c|c|c|c|c|c|c|}
\hline \multirow{3}{*}{$\begin{array}{l}\text { Ressources } \\
\text { idéologiques }\end{array}$} & \multicolumn{6}{|c|}{ SITUATION SOCIOPROFESSIONNELLE } & \multirow{3}{*}{ Total } \\
\hline & \multicolumn{2}{|c|}{ GUÉRISSEUR } & \multicolumn{2}{|c|}{ VENDEUR } & \multicolumn{2}{|c|}{ DÉMARCHEUR } & \\
\hline & Freq & $\%$ & Freq & $\%$ & Freq & $\%$ & \\
\hline La compétence & 6 & 14.63 & 17 & 41.46 & 18 & 43.90 & $41(100 \%)$ \\
\hline Le markéting social & 13 & 14.13 & 65 & 70.65 & 14 & 15.22 & $92(100 \%)$ \\
\hline L’innovation & 14 & 40.00 & 15 & 42.86 & 6 & 17.14 & $35(100 \%)$ \\
\hline La valorisation & 28 & 50.91 & 19 & 34.54 & 8 & 14.54 & $55(100 \%)$ \\
\hline Total & 61 & 27.35 & 116 & 52.02 & 46 & 20.63 & $\begin{array}{c}223 \\
(100 \%) \\
\end{array}$ \\
\hline \multirow[t]{2}{*}{$\mathrm{P}$ value } & & & & & & $<0,0001$ & \\
\hline & \multicolumn{3}{|c|}{ V de Cramer $=0,320$} & & 6ddl & & \\
\hline
\end{tabular}

Ces résultats montrent qu'il existe une différence significative entre les différentes ressources mobilisées par chaque acteur en fonction de sa situation professionnelle. En d'autres termes les guérisseurs privilégient la valorisation et l'innovation par rapport aux autres ressources idéologiques alors que les vendeurs de médicaments et les démarcheurs quant à eux privilégient respectivement le marketing social et la compétence. La comparaison des résultats montrent que la ressource idéologique la plus mobilisée par les jeunes guérisseurs est la valorisation (50.91\%). Les vendeurs de médicament quant à eux mobilisent deux ressources idéologiques dans les proportions suivantes : Marketing social (70.65\%) et l'innovation (42.86\%). Enfin ces résultats montrent également que la mobilisation de la compétence en tant que ressource idéologique est plus importante chez les démarcheurs soit (43.90\%).

\section{Discussion}

\section{La Mobilisation Du Capital Humain Comme Ressource Incontournable.}

Les données de l'enquête permettent de connaitre les caractéristiques socioprofessionnelles mobilisées par les jeunes déscolarisés pour leur insertion dans la médecine traditionnelle. Il y'a d'un côté les données en relation avec le choix professionnel et d'un autre côté les données en relation avec le capital humain qui désigne le niveau d'éducation et l'expérience professionnelle acquis par le travailleur au cours de sa vie 
professionnelle (Adiko et al. 2010 ; Zammar, 2012). Les résultats de l'étude montre que le niveau d'éducation des jeunes déscolarisés est acceptable (60.54\% ont le niveau primaire) avec des exceptions allant jusqu'au niveau supérieur (6.73\%). Cette situation constitue un atout fondamental pour les jeunes guérisseurs dans leur interaction avec les guérisseurs plus âgés. En effet pour éviter les mesures répressives imposées par les pouvoirs publics et entretenir une bonne image sociale dans un contexte de valorisation des médicaments traditionnels, les jeunes dynamiques peuvent servir d'allier. Une telle situation a pour avantage de favoriser une division des taches, ainsi qu'une coopération par la mise à la disposition des uns et des autres, soit de leur capital social, soit de leur capital intellectuel. Par conséquent, nous avons constaté que les jeunes proposent, dans la plus grande partie des cas, des documents écrits qui sont distribués aux clients; ainsi qu'aux passants dans la rue, dans les gars, aux automobilistes, à l'intérieur des moyens de transport en commun urbain comme interurbain, à tous heures, notamment dans les zones de grande affluence. Une telle approche, en plus de faire la promotion de la médecine traditionnelle, améliore l'activité des guérisseurs plus âgés. Il ressort de nos investigations, l'existence d'interaction entre guérisseurs plus âgés et jeunes déscolarisés qui usent de leurs niveaux d'instruction et de leur savoir-faire pour négocier des relations d'activité avec les plus âgés. Relativement au niveau d'instruction, les enquêtés mobilisent une autre dimension du capital humain. De fait, en vue de leur insertion dans l'activité de la médecine traditionnelle, les jeunes guérisseurs mobilisent l'expérience professionnelle acquise de leurs interactions avec les guérisseurs plus âgés. L'expérience ainsi acquis, ils prennent leur autonomie ou indépendance et s'installent pour leur compte. Le capital humain ainsi décrit favorise des rapports sociaux particuliers entre les jeunes guérisseurs et les plus âgés, en vue de leur insertion dans les activités de la médecine traditionnelle.

\section{De La Mobilisation Du Capital Social À L'insertion Des Jeunes Déscolarisés Dans La Traditherapie}

L'analyse des résultats de l'enquête et du contenu des sources documentaires, des informations recueillies auprès du Programme National de Promotion de la Médecine Traditionnelle et des guérisseurs visait à saisir le capital social mobilisé par les jeunes déscolarisés pour leur insertion dans la tradithérapie. Il ressort de celle-ci (l'analyse), que différents aspects du capital social sont mobilisés par les jeunes déscolarisés allant des associations, à l'accompagnement, au parrainage et à la collaboration entre guérisseurs, notamment avec les plus âgés. Ces informations nous permettent d'établir que les associations représentent la ressource la plus importante mobilisée par les jeunes guérisseurs, soit 70.78\%. D’après les informations 
rapportées auprès des enquêtés, cette situation est rendue possible par l'existence de plusieurs organisations associatives et non gouvernementales de la médecine traditionnelle dans le district d'Abidjan. Parmi celles-ci environ 23 ont un récépissé de déclaration contre 27 qui attendent encore le leur. En plus, il existe une relative collaboration entre acteurs de la médecine moderne et ceux de la médecine traditionnelle. Cela se traduit par la mise en place du projet pilote centre de médecine traditionnelle au sein du CHU de Treichville. Ces résultats sont en accord avec ceux de Manouan et al. (2010) qui ont montré dans leur étude réalisée dans le district d'Abidjan que 23\% des tradipraticiens déclarent collaborer avec la médecine moderne. Une telle situation est attestée, par les constats faits lors de nos investigations, notamment des propos de nos enquêtés. Ils soutiennent que depuis peu certains tradipraticiens se tournent vers les laboratoires d'analyse pour les tests de toxicité de quelques-uns de leurs médicaments. Aussi, n’hésitent-ils pas pour certains tradipraticiens à demander à leurs patients, selon le mal soupçonné, des analyses de laboratoires, pour la confirmation de leur diagnostic. Cependant, soulignons que cette mobilisation des ressources associatives par les jeunes guérisseurs n’est pas généralisable ; car l'étude d’Akoto et al. (2001) réalisée au Cameroun révèle que les jeunes autochtones ou originaires de la région de Bafia, qui déclarent exercer la médecine traditionnelle à titre principal, sont les plus réticents à devenir membres d'une association. Toutefois, cette étude souligne que les allogènes, qui semblent à la recherche d'une reconnaissance et d'une légitimité locale, sont les plus prompts à s'organiser en association, ce qui corrobore les résultats de notre étude. En effet, il ressort de l'analyse de nos investigations que les jeunes guérisseurs, qui ont le moins de possibilités ou de potentialités à revendiquer une légitimité traditionnelle, sont les plus favorables à la constitution d'associations et d'ONG de tradipraticiens reconnues officiellement.

\section{L'activation Des Ressources Idéologiques Comme Facteur D'insertion Socioprofessionnelle}

Les investigations réalisées auprès des enquêtés permettent d’établir une relation entre les ressources idéologiques mobilisées par les jeunes déscolarisés et leur situation socioprofessionnelle. Les données de cette étude montrent que les ressources idéologiques mobilisées par chaque enquêté sont fonction de sa profession. Les guérisseurs enquêtés citent majoritairement la « valorisation » comme ressources idéologiques mobilisées (50.19\%). Cette situation pourrait être due à en croire Abdmouleh (2009), à la recherche d'un moyen pour attirer le maximum de clients et conquérir les marchés urbains. C'est enfin un moyen pour sortir de la marginalité, éviter les mesures répressives et entretenir une bonne image sociale. Au nombre de ces 
mesures, on citer entre autres l'interdiction d'usage de la télévision et des radios nationales pour faire la publicité et l'interdiction de vendre les médicaments dans les moyens de transports en commun. Il est également relevé dans cette étude que la majorité des enquêtés (70.65\%) qui mobilisent le marketing comme moyen d'insertion sont des vendeurs de médicaments traditionnels. Soulignons également que parmi les enquêtés ayant cité majoritairement l'innovation comme ressource mobilisée pour leur insertion, figurent les vendeurs de médicament (42.46\%). Ces résultats confirment l'étude d'Abdoumouleh (2009), selon laquelle les guérisseurs se livrent à une véritable stratégie de marketing. En effet, ces guérisseurs ne se satisfont plus des procédures classiques (réseau d'amis et de clients). Ils usent de moyens plus modernes : tel que l'emploi des agents (publicitaires) répartis dans les régions avoisinantes (voire dans tout le pays) pour faire l'éloge de mérites et de dons des guérisseurs en question. On note enfin que les enquêtés qui ont cité majoritairement la compétence comme ressource idéologique sont les démarcheurs (43.90\%). Ces compétences générales, relatives au niveau d'instruction permettent aux jeunes guérisseurs dans leurs interactions avec les guérisseurs plus âgés de se définir une situation socio-professionnelle. À l'instar d’études antérieures (Mukandu, 2014 ; Kuwekita, 2009 ; Kuwekita et al., 2000), la nôtre établit également que le recours aux tradipraticiens est dû à leurs compétences reconnues ou supposées l’être.

\section{Conclusion}

La présente étude a permis dans un premier temps de définir les caractéristiques socioprofessionnelles et les ressources mobilisées par les jeunes déscolarisés pour leur insertion dans la tradithérapie. A l'issue de nos investigations, il ressort qu'ayant majoritairement le niveau primaire (60.54\%), et dans une moindre mesure le niveau secondaire (32,63\%), ces jeunes déscolarisés sont pour la plupart des vendeurs de médicaments traditionnels (42.46\%). Ils ont également en majeure partie une expérience professionnelle d'au moins 5 ans et plus (28.70\% et $37.22 \%$, soit $65.92 \%$, voir tableau $\left.\mathrm{n}^{\circ} 1\right)$. Aussi, la présence des jeunes déscolarisés dans l'activité de la médecine traditionnelle en Côte d’Ivoire résulte-t-elle de multiples ressources mobilisées par ceux-ci qui ne sont pas toujours aisées à élucider. Néanmoins, cet article nous a permis de cerner les ressources relationnelles et idéologiques qui sous-tendent les stratégies d'insertion des jeunes déscolarisés dans la tradithérapie. Autrement dit, de l’étude des interactions des jeunes guérisseurs avec leur environnement socioprofessionnel, il ressort que les ressources relationnelles et idéologiques se révèlent être des ressources capitales dans leur insertion dans la tradithérapie. Car la mobilisation de telles ressources comme stratégie d'insertion dans la tradithérapie, permet aux jeunes déscolarisés de négocier de nouveaux 
rapports avec les guérisseurs plus âgés. Ainsi, au nombre les stratégies qui sous-tendent l'insertion des jeunes déscolarisés dans la tradithérapie, les productions idéologiques se fondent autour de la compétence, du markéting social, de l'innovation et de la valorisation. Aussi l'étude révèle telle l'existence de quatre ressources relationnelles mobilisées par les jeunes déscolarisés pour leur insertion à savoir la collaboration ou coopération, l'accompagnement, le parrainage et les associations. En somme, ces ressources se révèlent, dans leur ensemble, des ressources stratégiques importantes, utilisées par ces jeunes déscolarisés, en vue de leur insertion dans la tradithérapie.

\section{References:}

1. Abdmouleh R (2009). Les guérisseurs et la quête d'une légitimité. Sociologie d'une profession illégitime. Comité national d'éthique médicale. Treizième Conférence Annuelle Sousse, le 4 décembre 2009.

2. Adiko A. F, Matthys B., Cissé G., Bonfoh B., Tanner M. et Jürg U. (2010). « Relation entre le capital humain des maraîchers urbains et leurs comportements de prévention des risques sanitaires sur les sites de culture à Abidjan (Côte d'Ivoire) » in [Vertigo].La revue électronique en sciences de l'environnement, vol. 10, n 2, 2010.

3. Akoto M.E., Songue B.P., Lamlenn S., Kemajou W. P.J. et Gruénais M-E (2010). «Infirmiers privés, tradipraticiens, accoucheuses traditionnelles à la campagne et à la ville ». In Bulletin de l'APAD, $n^{\circ} 21$

4. Coulibaly G.S. (2007). Evaluation de la collaboration entre la médecine moderne et la médecine traditionnelle dans la région du Sud Bandama, Thèse de médecine, Abidjan : UFR des sciences médicales, p.107

5. Groupe Ivoirien de Recherche sur l'Economie, la Société et la Culture Africaines (GIRESCA) (1995). Les représentations de la santé et de la maladie chez les ivoiriens (juillet 1994 - juillet 1995), Abidjan, Edition de l'OMS, p.380

6. Kisangau D.P., et al. (2007). « Use of traditional medecines in the management of HIV/AIDS opportunistic infections in Tanzania: a case in the Bukoba rural district». In J Ethnobiol Ethnomed, 3:29.

7. Manzambi K. J., Mbadu K. V., Bruyère Olivier1, Reginster J-Y (2009). «Les déterminants du comportement de recours au tradipraticien en milieu urbain africain: résultats d'une enquête de ménage menée à Kinshasa, Congo ». In Revue Psychologie \& Société Nouvelle 7 : 3-19. 
8. ManzambiK. J., Mbadu K. V., Bruyère O., Reginster J-Y (2013). Perception et Comportement de recours aux soins de santé dans les Pays en Voie de Développement. Le cas de la République Démocratique du Congo. Liège, Belgique. Les Éditions du Céfal, Liège.

9. Kroa E. (2000). Evaluation de l'efficacité du traitement traditionnel de l'accès palustre simple à Plasmodium falciparum à Ahougnanfoutou, département d'Agnibilékro, Thèse de médecine, Abidjan : UFR des sciences médicales.

10. Manouan N. J-M., N’Guessan B.B. Kroa E, Tiembré I. (2010). "Identification des acteurs de la médecine traditionnelle en Côte d'Ivoire : Cas du District Autonome d'Abidjan ». In Ethnopharmacologia, n²46, décembre 2010.

11. Pison G. (2009). "Un défi pour demain : le vieillissement démographique rapide des pays du sud ». In Réseau Canopé Idées économiques et sociales $\mathrm{N}^{\circ} 157$ pages 18 à 23. 